\title{
TATUAGEM E REALIDADE AUMENTADA: UM PROJETO DE DESIGN DE INTERAÇÃO BASEADO EM ARTEFATOS EPITELIAIS
}

Stephanie Martins Pinto da Costa

Universidade Federal de Juiz de Fora

stephaniempcosta@gmail.com

Jairo Calmon

Universidade Estadual de Feira de Santana

jairocalmon@ecomp.uefs.br

\author{
Angelo Loula \\ Universidade Estadual de Feira de Santana \\ angelocl@ecomp.uefs.br \\ Claudio Goes \\ Universidade Estadual de Feira de Santana \\ cegoes@gmail.com \\ João Queiroz \\ Universidade Federal de Juiz de Fora \\ queirozj@gmail.com
}

Resumo: Esse trabalho apresenta um sistema de Realidade Aumentada (RA) para a visualização de tatuagens na pele, que pode ser descrita como um espaço estruturado de artefatos (tatuagens) que funcionam como dispositivos epiteliais interativos. O sistema desenvolvido em RA gera imagens que acompanham a movimentação do corpo, deformações da pele, e são eficazes com oclusões parciais dos marcadores. De acordo com nossa abordagem, a pele não é apenas uma superfície capaz de desempenhar funções de proteção, absorção, controle e percepção de sensações obtidas a partir da interação com o ambiente. Sua natureza estendida atua como interface epitelial dinâmica, aberta à incorporação e acoplamento de novas tecnologias com propriedades semióticas interativas, inteligente e aumentada. Tornando-se um domínio de pesquisa em Design de Interação.

Palavras-chave: interface epitelial, realidade aumentada, tatuagem aumentada, design de interação

Abstract: This paper presents an Augmented Reality system (RA) for tattoos display on the skin, which can be described as a space structured by artifacts (tattoos), that act as interactive epithelial devices. The system developed in AR generates images that follow the movement of the body, skin deformation, and are effective with partial occlusions of the markers. According to our approach, the skin is not only a surface able to perform protection, absorption, control and perception of sensations derived from the interaction with the environment. The extended nature of the skin acts as epithelial dynamic interface, open to incorporation and coupling of new technologies with interactive, intelligent and augmented semiotic properties. Thus, the skin as epithelial interface (skin interface), becomes a search domain in Interaction Design. 
Keywords: skin interface, augmented reality, augmented tattoo, interaction design

\section{INTRODUÇÃO}

Pesquisas recentes em diversas áreas têm produzido tecnologias digitais e/ou bioinspiradas centradas no corpo (on-body technologies), como biossensores (JIA, 2013), nanotransistores (SINGER, 2009) (CHENG, 2015), nano-ILEDs (KIM, 2011), mecanismos de impressão de transistores de silício em materiais ultrafinos e flexíveis (KIM, 2009) (METTERS, 2011) (MA, 2011), para criação de novas modalidades de artefatos corpóreos e epiteliais, alterando de forma radical o papel semiótico da pele (BITARELLO et al. 2011) (BITARELLO \& QUEIROZ, 2014) (RANCK, 2012) (LIU, 2016). Tais artefatos, capazes de acoplamento epitelial (superficiais ou invasivos) (DUNNE, 2008) (LIU, 2016), reconcebem a pele como um espaço dinâmico e interativo, modificando o que conhecemos hoje como 'comunicação incorporada' (embodied communication). 0 uso de Realidade Aumentada (RA) fornece novas possibilidades de experimentação neste domínio. As implicações mais importantes acontecem em medicina (NICOLAU et al., 2005) (HORN, 2013), educação (MCMAHON, 2015), entretenimento (CASSINELLI et al., 2012) (MAURICIO et al., 2015), artes e design (BITARELLO \& QUEIROZ, 2014) (BITARELLO et al., 2011) (HAMDAN, 2016).

O propósito deste trabalho é apresentar um sistema de Realidade Aumentada (RA) para a visualização de tatuagens na superfície da pele. O sistema identifica a superfície da pele e suas deformações, e ajusta artefatos multimídias que acompanham tais deformações, mimetizando uma tatuagem real inscrita na pele. A próxima seção (seção 2) apresenta diversos sistemas de aplicação de RA envolvendo novas formas de tatuagens. Nesta seção, exploramos também algumas propriedades relacionadas à eficiência e robustez de processamento dos sistemas. Em seguida (seção 3), apresentamos uma análise dos resultados parciais já obtidos, e as considerações finais.

\section{TECNOLOGIA E TATUAGEM}

Projetos relacionados ao uso de Electronic Ink como E-ink tattoo (EMERGE INTERACTIVE, 2016), Changeable Tattoos (LEVY, 2001) e Glucose-monitoring Tattoo (SINGER, 2009) usam dispositivos eletrônicos e biomateriais que, quando acoplados, geram tatuagens com propriedades interativas (BITARELLO \& QUEIROZ, 2014). Nesses projetos, diversos fatores de vestibilidade (wearability factors) são cuidadosamente considerados para criação e desenvolvimento dos dispositivos em uma abordagem da pele como interface interativa (LIU, 2016) (VEGA, 2016).

\subsection{Tatuagem e Realidade Aumentada}

A Realidade Aumentada (RA) suplementa a realidade com informações virtuais geradas por computador em tempo real. Segundo Azuma (AZUMA, 2001), um sistema de RA (i) combina objetos reais e virtuais no espaço físico real, (ii) é interativo e em tempo real, e (iii) fornece uma visualização de objetos reais e virtuais interligados. Assim, uma tatuagem gerada por RA é um objeto virtual, modelado por computador, 
que atua com entidades reais, em tempo real, a partir de uma visualização direta ou indireta do mundo físico (Figura 1).

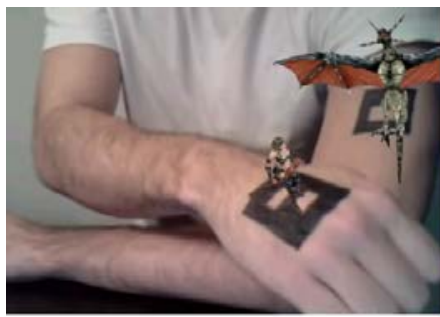

(a)

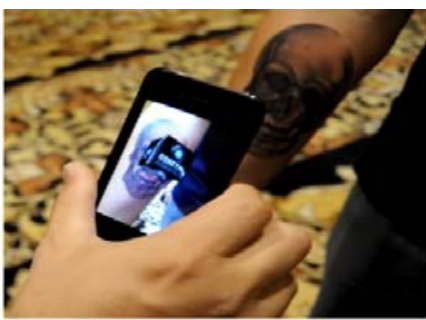

(b)

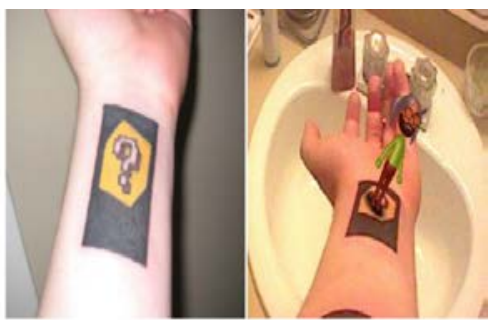

(c)

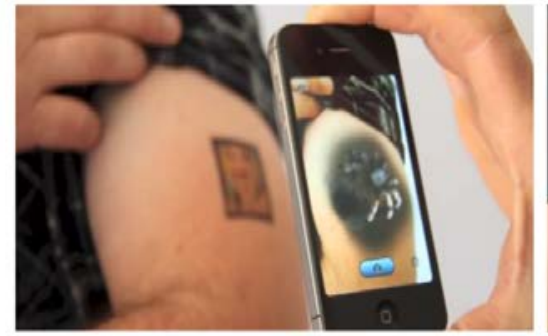

(d)

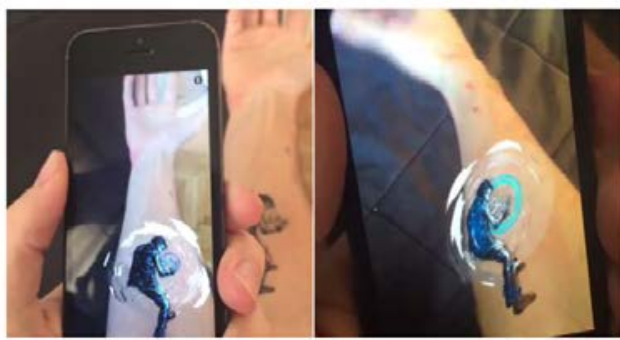

(e)

Figura 1 - (a) ThinkAnApp -- a posição do marcador tatuado na pele é obtida; surgem um dragão e uma guerreira 3D animados. (b) caveira. (c) marcador do Nintendo DS e personagem 3D. (d) aranha em animação. (e) personagem de HQ reconhecido como marcador -- imagem animada sobreposta. Fonte: https://www.youtube.com/watch?v=YD_C2lc3c3Y. Em (b, d, e), aplicação em dispositivo móvel (smartphones). Adaptada de (CALMON, 2015).

\subsection{Tatuagem aumentada (Augmented Tattoo)}

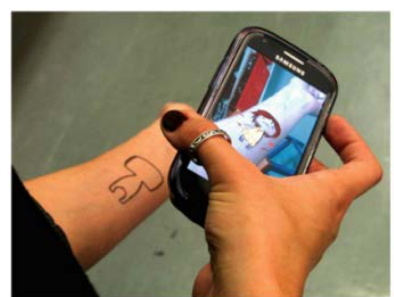

(a)

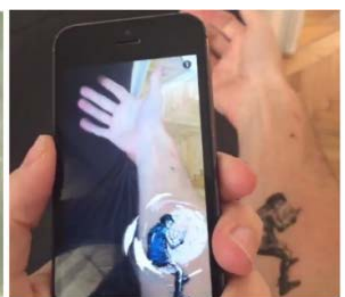

(b)

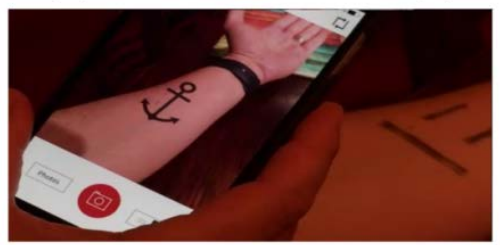

(c)

Figura 2 - Tatuagens geradas por Realidade aumentada. (a) TattooAR.

Fonte: (MAURICIO et al., 2015) (b) Modern Polaxis Tattoo. Fonte:

https://www.youtube.com/watch?v=YD_C2Ic3c3Y. (c) Ink Hunter. Fonte: http://www.ny1.com/nyc/allboroughs/app-wrap/2016/04/8/app-wrap---tunity----ink-hunter--.html.

A Tatuagem Aumentada (TA) (Figura 2) permite a criação de artefatos dinâmicos, interativos e multimodais, por meio de imagens 2D, 3D, animações, vídeos, efeitos sonoros e hápticos. A pele passa a desempenhar um papel de interface epitelial com características responsivas resultantes da relação entre sujeito (tatuado), objeto (TA) e ambiente. 
Em TattooAR (MAURICIO et al., 2015) são apresentados dispositivos computacionais, em que um marcador da aplicação de RA, de material transparente, é colocado sobre a superfície da pele fornecendo informação para sobreposição da tatuagem aumentada. Nessa aplicação, o marcador não é apagado, não são detectadas as deformações da pele, nem há tratamento de possíveis oclusões do marcador utilizado, de modo que, se um objeto for interposto entre o marcador e a câmera, o sistema não é capaz de gerar a tatuagem. Em Modern Polaxis Tattoo (SUTU, 2014), a própria tatuagem é detectada como marcador e a imagem da tatuagem original é transformada em uma animação com efeitos sonoros desenvolvida em Flash e integrada a RA, que segue a movimentação do corpo. Em Ink Hunter (ROHACHOVA, 2016), a aplicação fornece uma visualização de tatuagem por meio de RA para auxiliar na decisão de uma inscrição tradicional na pele. Nesse sistema, um marcador de três traços é desenhado e os processos computacionais empregados apresentam um grau de detecção dos limites da pele na imagem. Porém, as imagens não acompanham a superfície da pele para realizar a sobreposição da TA, usando técnicas de recorte para que ela não ultrapasse a área delimitada de pele.

Esses trabalhos (MAURICIO et al., 2015), projetos comerciais (SUTU, 2014) e aplicativos para dispositivos móveis (ROHACHOVA, 2016) (DYBWAD, 2010), abordam a pele como suporte para aplicação de RA. Entretanto, poucos trabalhos têm atuado de modo a considerar a pele como uma superfície interativa. Em nossa proposta, são identificadas as deformações da pele, de modo a ajustar artefatos multimídias que acompanham tais deformações, mimetizando uma tatuagem real (CALMON et al., 2014) (CALMON et al., 2015) (CALMON, 2015). Para isso, são investigados diversos fatores de vestibilidade no contexto da pele como interface epitelial - (i) localização (regiões aptas aos marcadores), (ii) movimentação e características da pele (tonalidade, presença de pelos e marcas, tipos de movimentos naquela região), (iii) métodos de acoplamento (marcador na/sobre a pele), (iv) acessibilidade (visual, auditiva, tátil ou cinestésica no corpo), (v) interação (sistema-usuário), (vi) estético (formato do marcador em relação à região de acoplamento e criação da TA), (vii) isolamento (o material usado nos marcadores não pode interferir no desempenho do corpo), (viii) conexão e comunicação (informações capturadas, transferência das informações e uso pelos dispositivos do sistema), (ix) manutenção (marcadores e dispositivos do sistema).

A pele recebe um conjunto de marcadores discretos, superficiais, não invasivos, e manuscritos através de canetas comuns, ou corretivo líquido, inscritos em qualquer região do corpo, e removíveis (Figura 3).

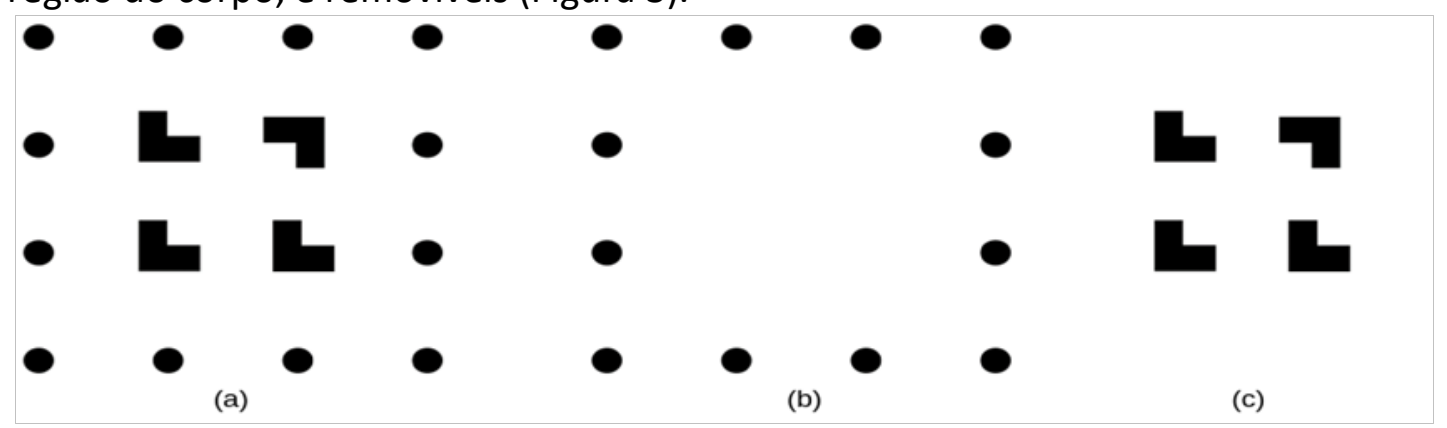

Figura 3 - (a) Conjunto de marcadores utilizados no sistema. Esse conjunto é formado por (b) marcadores periféricos circulares para fornecer informações de contorno e (c) marcadores especiais no formato de "L" (sólido) para rastreamento. Fonte: Adaptada de (CALMON, 2015) 

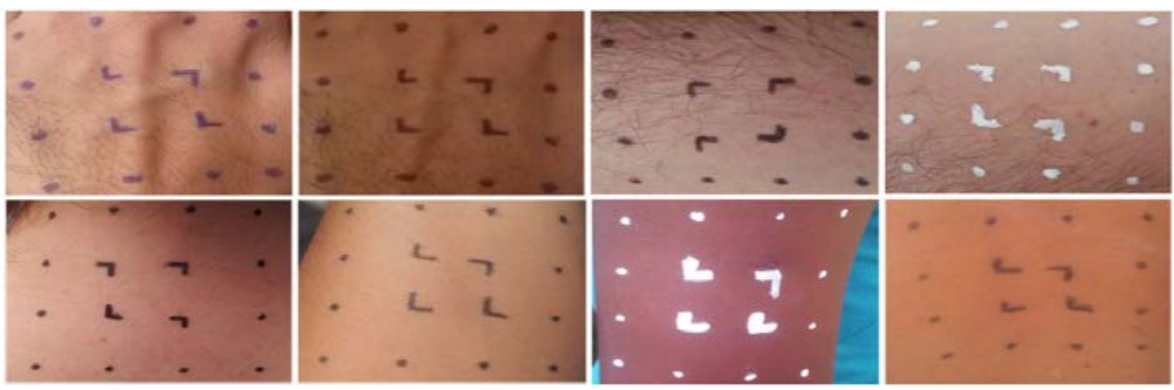

Figura 4 - Dataset de amostras close-ups de um conjunto de amostras de pele com características diferentes e o conjunto de marcadores feitos com materiais diferentes.

Fonte: Adaptado de (CALMON, 2015).

Após a inscrição dos marcadores (Figura 4), imagens e vídeos são capturados por câmeras monoculares comuns (câmeras de celular, tablets, Ipads, computadores) e, através de técnicas de registro $2 \mathrm{D}$, são detectados e identificados os marcadores (Figuras 5).

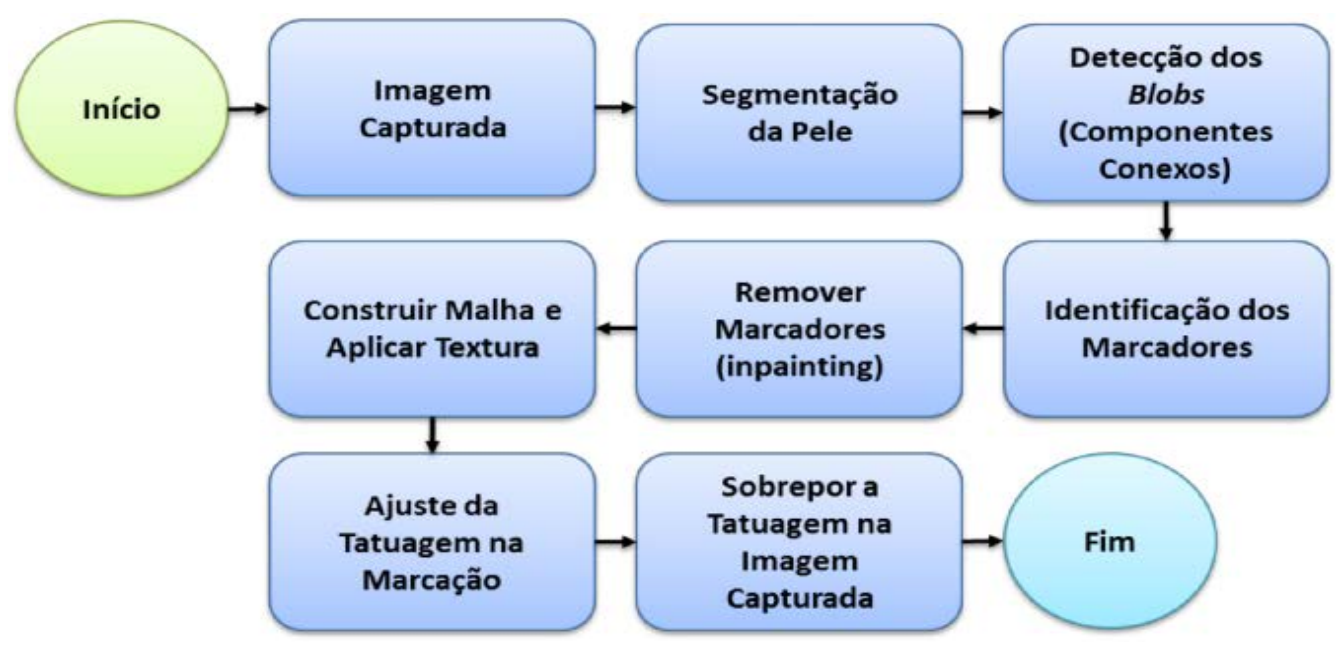

Figura 5 - Fluxo de ações executadas no sistema.

Fonte: Adaptada de (CALMON et al., 2014)

Através da análise da imagem adquirida de pele com marcador (Figura 6), é feita uma busca de informações para a segmentação da pele (Figura 7), preservação da textura, identificação dos marcadores centrais, e periféricos, e gerenciamento de possíveis oclusões desses marcadores. Com base nas informações adquiridas pela posição dos marcadores, é construída e renderizada uma malha com a tatuagem em imagem separada. Uma máscara de pele é utilizada para não renderizar as partes que não estão sobre a pele. Os marcadores são removidos da imagem original capturada e substituídos pela textura da pele (Figura 8). A imagem da tatuagem (Figura 9) editada pela máscara de pele é sobreposta à imagem original após o processo de remoção dos marcadores. Realizado esse processo, a imagem encontra-se pronta para ser exibida (Figura 10). O processo é realizado de forma a gerar a TA em tempo real. A tatuagem gerada é capaz de acompanhar a movimentação do corpo, possíveis deformações da pele, no momento em que elas acontecem, e oclusões de até quatro marcadores, devido ao posicionamento da superfície da pele em relação à câmera ou algum objeto 
interposto entre a pele e a câmera (Figura 11).

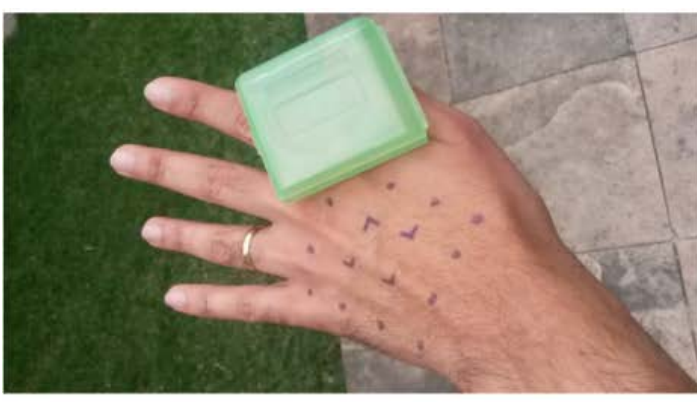

(a)

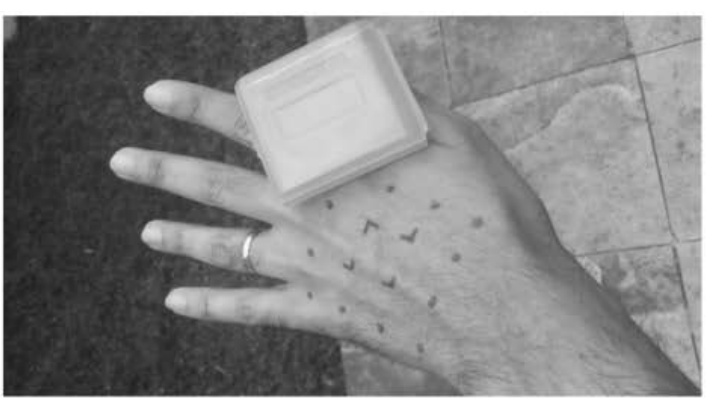

(b)

Figura 6 - Funcionamento do sistema. (a) Imagem capturada com oclusão parcial (b) Imagem em escala de cinza. Fonte: (CALMON, 2015)

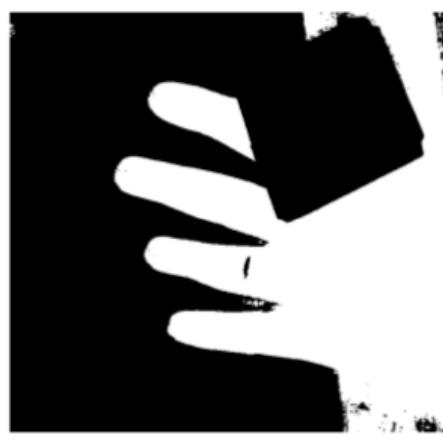

(a)

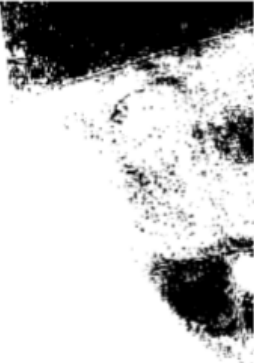

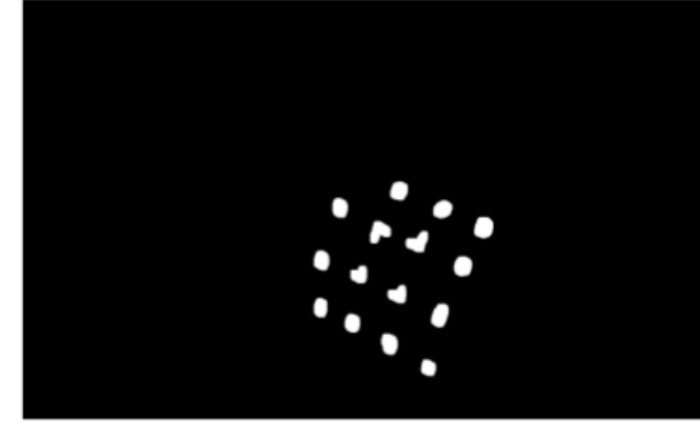

(b)

Figura 7 - Funcionamento do sistema. (a) Resultado da segmentação da pele com oclusão parcial (b) Marcadores detectados e isolados. Fonte: (CALMON, 2015)

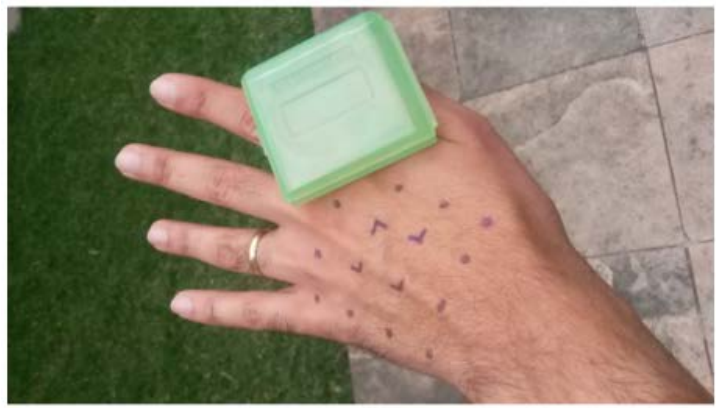

(a)

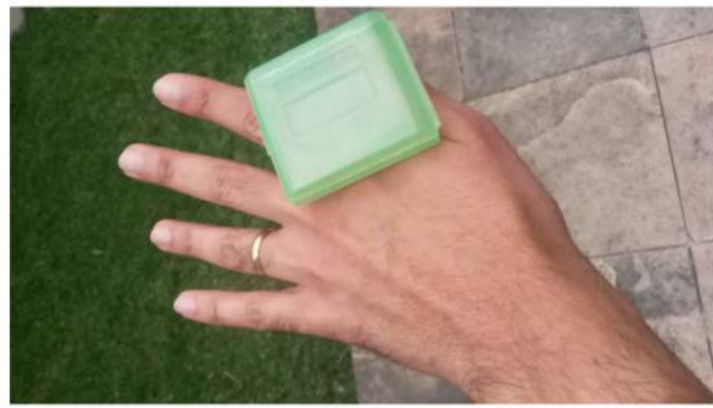

(b)

Figura 8 - Funcionamento do sistema. (a) Imagem original com oclusão parcial antes do processo de inpainting (processo de remoção dos marcadores) (b) Imagem após a aplicação do inpainting.

Fonte: (CALMON, 2015)

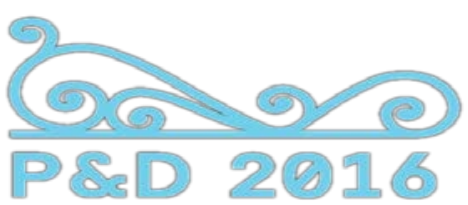




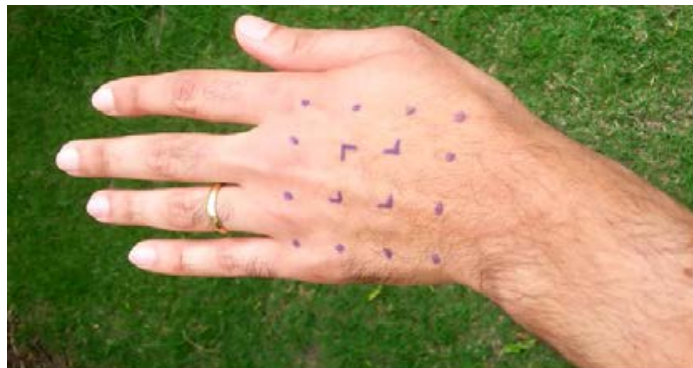

(a)

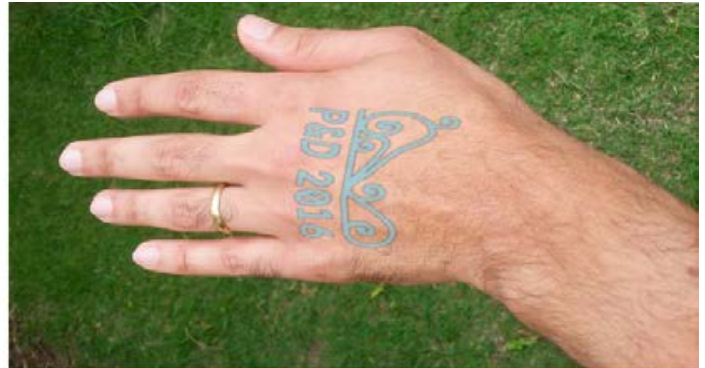

(b)

Figura 9 - Imagem de tatuagem a ser sobreposta na pele. Fonte: Adaptada de (http://www.ped2016.com.br/).
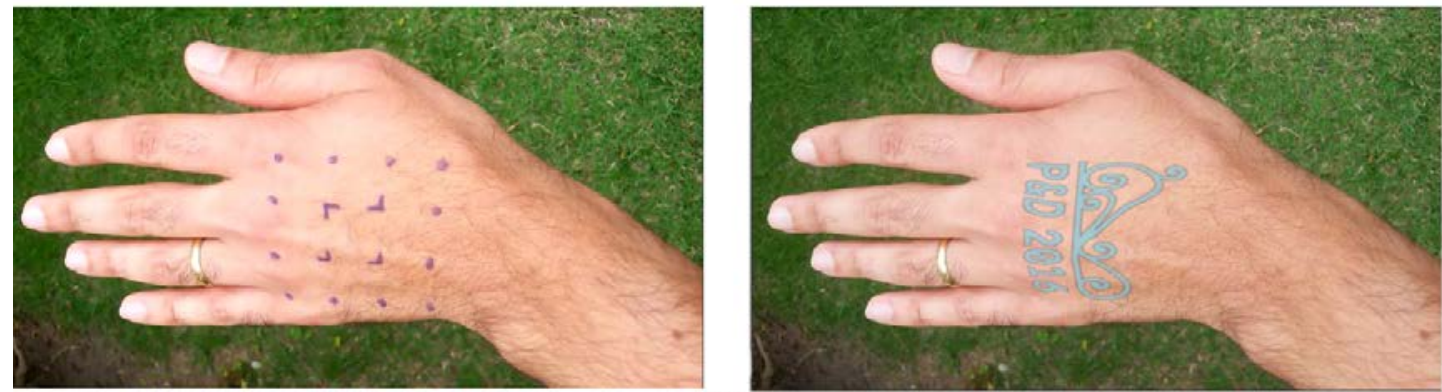

(a)
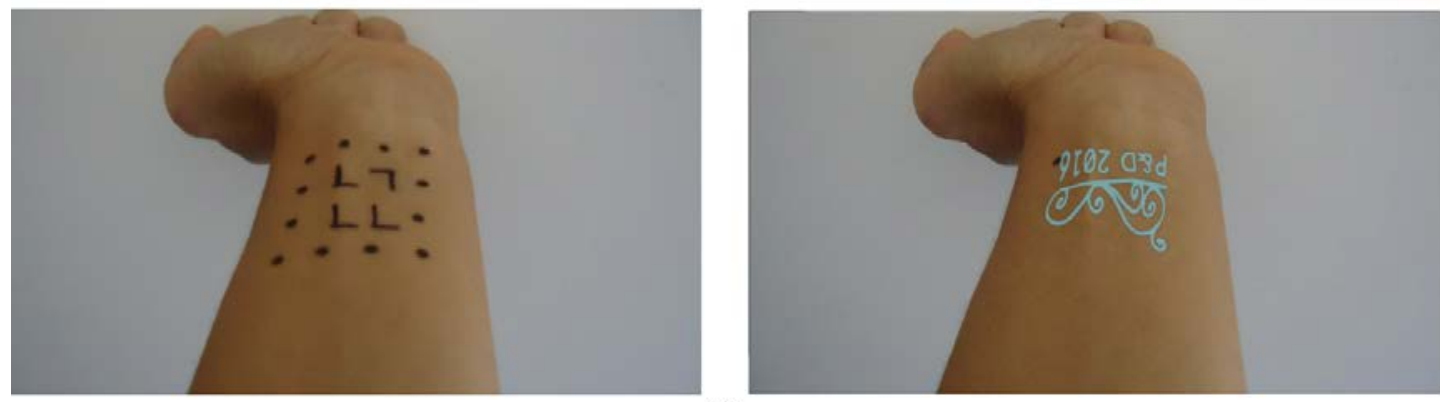

(b)
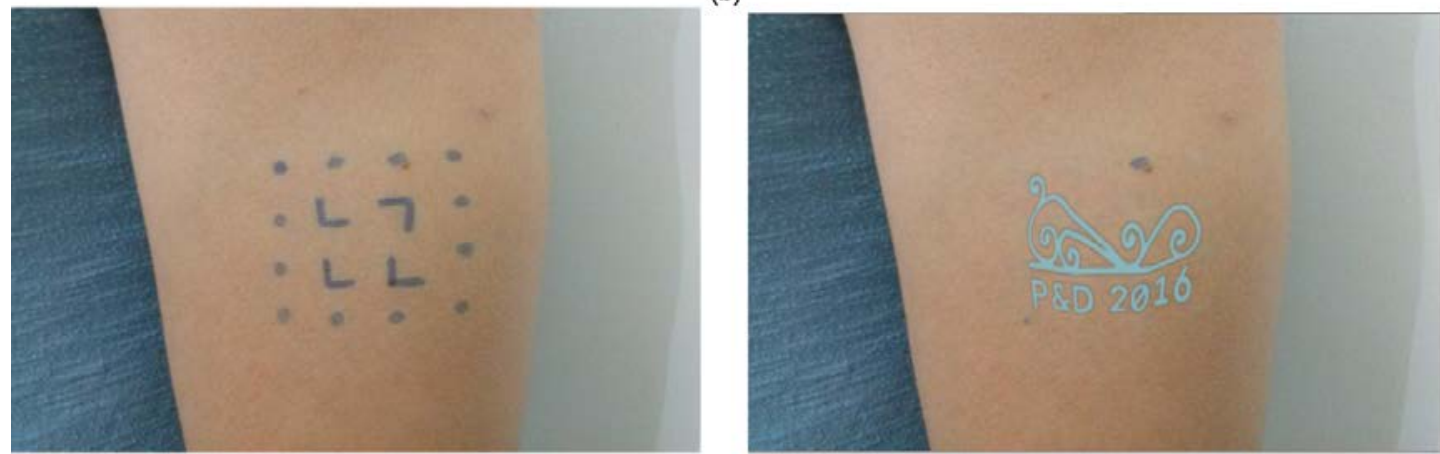

(c)

Figura 10 - Funcionamento do sistema. (a) Imagem original capturada sem oclusão. (b) Imagem com a tatuagem aumentada. Elaborada pela autora com base nas pesquisas realizadas.

Figura 11 - Conjunto de diferentes amostras de pele com variações de ponto de vista (distância e inclinação), movimentação, condições de iluminação e fundo. (a) lluminação natural, fundo não uniforme e mão com leve inclinação. (b) Iluminação não natural e fundo uniforme; falha ao não apagar um marcador. Mas ocorre o ajuste da tatuagem diante das deformações capturadas (c) lluminação não natural e fundo não uniforme. Nesse teste, o sistema não apaga um marcador devido a presença de uma pinta próxima a um dos marcadores. Elaborada pela autora com base nas pesquisas realizadas. 


\section{CONCLUSÃO}

O sistema desenvolvido (CALMON et al., 2014) (CALMON et al., 2015)(CALMON, 2015), projetado inicialmente para ambiente desktop, apresentou desempenho satisfatório em tempo real na visualização da tatuagem aumentada. Porque obtém um modelo de superfície da pele por meio de uma grade de pequenos marcadores (marcadores circulares periféricos e marcadores especiais centrais), o máximo de informação de pele é capturada e conservada; isto é, os marcadores não escondem a área de pele a ser sobreposta pela tatuagem. Ao contrário, quanto maior for a área de pele junto ao marcador, maior será a informação de pele capturada e conservada. 0 sistema apresentou uma performance satisfatória para a detecção dos marcadores com poucas imagens com problemas de renderização (Figura 11). Na análise de oclusões, o sistema também apresentou desempenho eficiente, tanto nos casos em que a oclusão resultou da própria superfície da pele relativa ao posicionamento da câmera, quanto com oclusão de um componente interposto entre pele e câmera. Alguns tipos de distorções, resultantes da modificação da posição da câmera em relação aos marcadores, também foram avaliados. Nessa análise, pequenas distorções de perspectiva dificultaram o processo de deteç̧ão dos marcadores. Contudo, nas situações de deformação na superfície da pele, o sistema apresentou robustez na detecção de marcadores, com deformações em diversas superfícies de pele. Outra situação de desempenho favorável ocorreu em condições adversas de iluminação. Ambientes com pouca iluminação, por exemplo, tendem a causar borramentos (motion blur) na imagem como resultado da tentativa de compensação automática realizada pela câmera. Nessas situações, o desempenho do sistema foi satisfatório apresentando a tatuagem aumentada como uma inscrição real.

De acordo com a abordagem desenvolvida aqui, a pele não é apenas uma superfície capaz de desempenhar funções de proteção, absorção, controle e percepção de sensações obtidas a partir da interação com o ambiente (JABLONSKI, 2013) (LIU, 2016). Sua natureza estendida atua como interface epitelial dinâmica (LIU, 2016), aberta à incorporação e acoplamento de novas tecnologias (PREESTER, 2010) (LIU, 2016) com propriedades semióticas interativas, inteligente (body intelligent) e aumentada (PREESTER, 2010) (VEGA, 2014) (LIU, 2016). Ao tratar a pele como interface epitelial (skin interface), capaz de acoplamento de dispositivos multimídias de RA, ela se torna um domínio de pesquisa em Design de Interação.

\section{REFERÊNCIAS}

ARGILE, Michael. Bodily Communication. New York: Methue, 1988.

ASCOTT, Roy. Reframing Consciousness -- Art, mind and technology. Intellect Ltd, 1999.

AZUMA, Ronald. Survey on Recent advances in augmented reality. IEEE Computer Graphics and Applications, 2001.

BITARELLO, Breno; FUKS, Hugo; QUEIROZ, João. New technologies for dynamic tattoo art. Proceedings of the fifth international conference on Tangible, embedded, and embodied interaction, 2011. P. $313-316$.

BITARELLO, Breno; QUEIROZ, João. Embodied semiotic artefacts: On the role of the 
skin as a semiotic niche. Technoetic Arts: A journal of speculative research. Volume 12. Number 1, 2014.

CALMON, Jairo; LOULA, Angelo; GOES, Claudio; QUEIROZ, João. Augmented Tattoo: a proposal of tattoo visualization in augmented reality. $X$ Workshop de Visão Computacional, 2014.

CALMON, Jairo; LOULA, Angelo; GOES, Claudio; QUEIROZ, João. Augmented Tattoo: Evaluation of an Augmented Reality System for Tattoo Visualization. $28^{\text {th }}$ SIBGRAPI Conference on Graphics, Patterns and Images, 2015.

CALMON, Jairo. Sistema de realidade aumentada para visualização de tatuagens. 2015. 94 f. Dissertação (Mestrado em Ciência da Computação) apresentada ao Mestrado como requisito parcial para obtenção do grau de Mestre em Ciência da Computação. Universidade Federal da Bahia e Universidade Estadual de Feira de Santana. Mestrado Multi-Institucional em Ciência da Computação.

CHENG, Q.; PENG, Z.; LIN, J.; Li, S.; WANG, F. Energy haversting from human motion for wearables devices. Nano/Micro Engineered and Molecular Systems, IEEE 10th International Conference on, (2015), 2015. P. 409-412.

CASSINELLI, A. et al. Skin games. Proceedings of the 2012 ACM international conference on Interactive tabletops and surfaces - ITS'12, ACM Press, New York, New York, USA, p.323, 2012. Disponível em:

https://dl.acm.org/citation.cfm?doid=2396636.2396690. Acesso em 21 de abril de 2016.

DUNNE, L. Wearability in wearables computers. Wearable Computers, 2008. ISWC 2008. 12th IEEE International Symposium on (2008), 2008. P. 125.

DYBWAD, B. Augmented Reality Tattoo Makes Your Skin Come Alive. Disponível em: http://mashable.com/2010/02/17/augmented-reality-tattoo/. Acesso em: 14 de maio de 2016.

Emerge interactive. Disponível em:

http://www.emergeinteractive.com/insights/detail/introducing-the-worlds-firstelectronic-ink-tattoo. Acesso em: 4 de junho de 2016

FURHT, Borko. Handbook of augmented reality. Springer Science \& Business Media, 2011. 746 p.

HAMDAN, Camila. Conceitos e Contextos da Realidade Aumentada Móvel: Corpo, Arte e Tecnologia. IV Simpósio Internacional de inovação em mídias interativas. Universidade Federal de Goiás, 2016.

HARMON, K. Skinlike electronic patch takes pulse, promises new human-machine Integration, 2011. Disponível em: https://www.scientificamerican.com/article/skinelectronic-patch/. Acesso em: 22 de abril de 2016.

HORN, M. A Look into the Body - Augmented Reality in Computer Aided Surgery, 2013. Disponível em: http://www.in.tum.de/en/research/researchhighlights/augmented-reality-in-medicine.html. Acesso em: 9 de junho de 2016. 
KIM, D.; LU, N.; MA, R. et al. Epidermal electronics. Science, 333: 6044, 2011. p. 838 43.

KIM, D.; KIM, Y.; AMSDEN, J. et al. Silicon electronics on silk as a path to bioresorbable, implantable devices. Applied Physics Letters, 95: 13, 2009.P. 133701 03.

LEVY, D.; CHERRY, J. Changeable Tattoos, U.S. Pat. 6,192,890, 2001.

JIA, W.; Bandodkar, A. J. et al. Electrochemical tattoo biosensors for real - time noninvasive lactate monitoring in human perspiration. Anal Chemistry, 85: 14, 2013. P. 6553 - 60.

LIU, X., Vega, K. Wearability Factors for Skin Interfaces, 2016.

MANNING, Erin. RELATIONS CAPES: Movement, Art, Philosophy. The MIT Press: Cambridge, Massachusetts, 2009.

MA, Z. An electronic second skin. Science, 333:6044, 2011. P. 830-31.

MAURICIO, Gabriela S.; BONELLI, João S.; CHAGAS, Maria G. TattooAR: Augmented Reality Interactive Tattoos. DUXU 2015, Part II, LNCS 9187. Springer International Publishing Switzerland, 2015. P. 667-674.

MCMAHON, Don D.; CIHAK, David F.; WRIGHT, Rachel E.; BELL, Sherry Mee. Augmented Reality for Teaching Science Vocabulary to Postsecondary Education Students With Intellectual Disabilities and Autism. Journal of Research on Technology in Education, 2015.

METTERS, J. P.; Kadara, Ro; Banks, Ce. New directions in screen printed electroanalytical sensors: An overview of recent developments. Analyst, 136:6, 2011. P. 1067-76.

SUTU, MODERN POLAXIS. Disponível em: http://modernpolaxis.com/. Acesso em 15 de maio de 2016.

NICOLAU, S. et al. A complete augmented reality guidance system for liver punctures: First clinical evaluation. Medical Image Computing and Computer - Assisted Intervention. LNCS 3749. Springer, 2005. P. 539 - 547, 2005. Disponível em: https://link.springer.com/chapter/10.1007/11566465_67. Acesso em 21 de abril de 2016.

PREESTER, Helena. Technology and the Body: the (Im) Possibilities of Reembodiment. Found Sci. Springer, 2010.

PENTLAND, A.P. Wearable intelligence. Scientific American, Incorporated, 1998.

RANCK, J. The weareable computing market: a global analysis. 2012.

REKIMOTO, J. (2001) GestureWrist and GesturePad: Unobstrusive wearable interaction devices. Wearable Computers, 2001. Proceedings. Fifth International Symposium on, (2001), 2001. P. 21 - 27.

ROHACHOVA, O.; RAZUMOVSKYI, P.; PETRUNKO, I.; PONOMARENKO, O. INK HUNTER. Disponível em: http://inkhunter.tattoo/. Acesso em: 9 de junho de 2016. 
RUMBLE VIRAL. Disponível em: https://www.youtube.com/watch?v=YD_C2Ic3c3Y Acesso em: 15 de maio de 2016.

SINGER, Emily. The Glucose-Monitoring Tattoo: A novel nanosensor could be used for skin-based glucose sensing. MIT Technology Review, 26 January, 2009. Disponível em: https://www.technologyreview.com/s/411770/the-glucose-monitoring-tattoo/. Acesso em: 22 de abril de 2016.

VEGA, Katia; FUKS, Hugo. Beauty Technology: Body Surface Computing. Computer 47, 4, 2014. P. $71-75$.

VEGA, Katia; FUKS, Hugo. Beauty Technology: Skin Interfaces. Human Computer Interaction Series. Springer, 2016. 